\title{
Género y espacialidad: análisis de factores que condicionan la equidad en el espacio público urbano*
}

\author{
Gender and Space: Analysis of Factors Conditioning \\ Equity in Urban Public Space
}

Recibido: junio 25 de 2010 ～Revisado: diciembre 3 de 2010 | Aceptado: enero 17 de 2011

\author{
PABLO PÁRAMO** \\ Universidad Pedagógica Nacional, Bogotá, Colombia \\ Andrea Milena Burbano Arroyo ${ }^{* * *}$ \\ Universidad Pedagógica Nacional y Universidad \\ Piloto de Colombia
}

Para citar este artículo. Páramo, P., \& Burbano, A. M. (2011). Género y espacialidad: análisis de factores que condicionan la equidad en el espacio público urbano. Universitas Psychologica, 10(1), 61-70.

\footnotetext{
* Naturaleza del artículo: Ensayo..

** Calle149No.54A-79 Bogotá.E-mail: pdeparamo@ gmail.com

**** Carrera 49 № 166-63 Bogotá. E-mail: arqmilenaburbano@gmail.com
}

\section{RESUMEN}

Este artículo discute la investigación sobre género en el espacio público, a través de tres perspectivas diferentes: las representaciones sociales y usos diferenciados del espacio, la división de roles en los espacios públicos y privados, y la planeación urbana del espacio. El trabajo recoge y analiza algunos estudios que complementan el estado del arte y la literatura sobre las mujeres y el espacio, en los que se da evidencia sobre la manera como las mujeres han sido segregadas del espacio público y son víctimas de las desigualdades de género. Ni el espacio público ni el género existen de forma absoluta, por el contrario, ambas categorías son construidas por el orden social y reproducidas por las prácticas sociales. Por último, se dan algunas recomendaciones para la planeación y la investigación, con el fin de contribuir a dar respuesta a las necesidades de las mujeres en el espacio público. Palabras clave autores

Género, espacio público, feminismo.

Palabras clave descriptores

Género, espacio público, feminismo y arquitectura.

\section{A B S T R A C T}

This article discusses gender research in urban public space through three different perspectives: the social representations and differentiated uses of space, the division of roles in public and private spaces, and urban planning of public space. The paper gathers and analyses some studies that complement the state of art and literature on women and space giving evidence on how women have been segregated from public space and are victim of gender inequalities. Public space does not exist absolutely nor gender; instead both are socially constructed by social order and reproduced by social practices. Finally, some suggestions for urban planning and research are given in order to respond women's needs in public space.

Key words authors

Gender, Public Space, Feminism.

Key words plus

Gender, Public Space, Feminism And Architecture. 


\section{Introducción}

La manera como se conforma el espacio de las ciudades, lo planificamos y hacemos uso de éste, refleja nuestra concepción de sociedad, reproduciendo la visión que tenemos de ella. Bajo este supuesto, el espacio no existe en forma absoluta sino relativa, resultado de una producción social y como una manifestación del orden social. El espacio reproduce el orden de valores de una sociedad, las clases sociales, la concepción que se tenga de familia y del papel que juegan las mujeres y los hombres en tal sociedad. Sin duda la relación entre el cuerpo y el espacio, sus manifestaciones y problemas, han encontrado su expresión en la arquitectura y en la planificación urbana de las ciudades (Sennet, 2003).

Los estudiosos del género exploran la manera como las mujeres y los hombres asumen comportamientos y actitudes diferentes, para relacionarse con y en el espacio. Dichas diferencias varían histórica y culturalmente de acuerdo a la edad, la clase social, la religión, entre otras circunstancias. En este contexto resulta importante analizar no solo el desarrollo investigativo reciente sobre la manera como distintos factores culturales condicionan la relación de la mujer con el espacio público, sino las razones por las cuales se observan estas formas de comportamiento diferenciadas frente a los hombres.

\section{Lo masculino y lo femenino en el espacio}

Según Franck (2002), la investigación feminista ha enmarcado el campo de la mujer en dos perspectivas: en primer lugar, en la discusión entre género y sexo, dentro de la cual cobra mayor importancia la primera categoría más que la segunda; el género es visto como un constructo social, sobrepuesto a la biología del sexo. Esta aproximación ataca la suposición esencialista de que la condición de mujer está estrictamente determinada por la biología; una creencia que estereotipa a las mujeres, que no respeta sus elecciones y no las representa adecuadamente dentro de la sociedad y la cultura. Y en segundo lugar, en la perspectiva de las experiencias y las necesidades de las mujeres, las cuales están condicionadas a su edad, orientación sexual, lugar de residencia, y muchas otras circunstancias individuales y culturales entre las cuales se encuentra la utilización del espacio.

Este artículo invita a la reflexión sobre la relaciones entre género y especialidad, a partir de tres áreas de investigación que, desde nuestra perspectiva, han orientado el campo de estudio: la identificación de representaciones diferenciales del ambiente a partir del género, la división entre lo público y lo privado en los roles masculino y femenino, y las formas de planificar y diseñar el ambiente urbano.

Los estudios sobre la espacialidad y el género dan evidencia de que existen diferencias importantes en las representaciones mentales y usos diferenciales del espacio. Saegert y Winkel (1980) por ejemplo, identifican roles masculinos y femeninos dentro del hogar, a partir de la manera como los objetos que existen dentro de la casa expresan la personalidad de las mujeres, mediante objetos de contemplación como las fotografías de familiares, de amigos queridos o de esculturas, más que representar la personalidad de sus esposos, quienes valoran en mayor medida los objetos de acción tales como los televisores, los equipos de estéreo, los equipos deportivos o los vehículos. A partir de esta diferenciación, se infiere que para los hombres el mundo es lugar para hacer cosas, mientras que para las mujeres es un lugar para relacionarse con las cosas, lo que no significa necesariamente que las mujeres no hagan cosas distintas.

Al mirar la manera como ocurre el proceso de socialización y de la construcción social del género en relación con la especialidad, los estudios muestran que a las niñas se les motiva para ser menos exploratorias, más temerosas y menos activas físicamente que los niños. El juego de las niñas involucra menos actividades de manipulación del ambiente y están más restringidas en su ocupación corporal del espacio (Young, 1990). Los manuales de etiqueta del siglo XIX establecían, para las adolescentes, que para las reuniones sociales la posición corporal era determinante de la imagen que trasmitía una mujer: " el cuerpo debe estar en posición natural, 
descansando los pies en el suelo, las piernas no deberían estar muy unidas, ni separadas, el vestido que cubra el pie hasta el zapato, evitar hacer movimientos que molesten la vista de los demás y toda familiaridad con quienes las visitan para evitar que les falten el respeto" (Aristizábal, 2007, p. 227).

Por otra parte, la diferenciación entre géneros surge como producto de la asignación del espacio público a los hombres y de la marginación de la mujer a los espacios privados o interiores (Folguera, 1982; Loyd, 1975). Históricamente los espacios de las mujeres han sido equiparados con espacios privados, siendo los espacios públicos el espacio de los hombres. En este sentido, hay una oposición simbólica entre la casa y el resto del mundo; lo femenino es la esfera opuesta a lo masculino que corresponde a la vida pública, de tal manera, que el rol de la mujer se asocia principalmente con la casa (Alarcón, 2007; Páramo \& Cuervo, 2006). Con la revolución industrial se generó una mayor disociación entre el lugar de trabajo y el de vivienda, asignando al hombre las funciones productivas y a la mujer las de la crianza y de mantenimiento del hogar, produciendo una segregación de funciones en el espacio. Si el lugar de residencia de la mujer es la casa, con seguridad hay factores que moldean el diseño y uso de esos espacios para hacerlos significativos y comprensibles, desde los patrones sociales que se le imponen a la mujer. Pero esto no fue solo producto de la revolución industrial en Inglaterra, ya que hay muchas culturas que han segregado los espacios domésticos a partir del género desde la antigua Grecia. A través de la historia, el género ha sido un determinante de actividades y actores, en los lugares públicos; los lugares públicos y la vida del dominio público como el género en sí mismo se entienden en relación a su antítesis, espacio privado y vida personal: la vida privada tiene sentido únicamente en relación a la vida pública (Prost \& Vincents, 1991). La configuración física, es decir, los arreglos espaciales que incluían el diseño de las ciudades, de los pueblos, las tiendas, las oficinas y los hogares, moldean el acceso de los individuos al conocimiento. Los espacios domésticos, aunque son sinónimos de espacios privados, han sido concebidos como los que suministran los límites que distinguen entre lo público y lo privado. Las actividades en los lugares públicos y el conocimiento del dominio público determinan el estatus, el poder, la naturaleza e igualdad de las relaciones interpersonales. Por esto es que para las feministas, la casa es vista como un mecanismo de domesticación de la mujer y, de esta manera, se intenta de situar a las mujeres en condición de inferioridad. Como lo argumenta Sonnet (1993), la historia ha mostrado que, en las ciudades, la casa ha sido donde las mujeres han aprendido a desempeñar las labores cotidianas de una madre de familia, que tienen que ver con la cocina, los cuidados de los hijos menores, la conservación de la ropa blanca y de la vestimenta de la casa, el manejo del hilo, las agujas, la lana, los tejidos. Aunque en el campo, se sumaban otras labores además de estas, como el cuidado de las aves y la pesquería. En este sentido, la casa se consideró un centro de aprendizaje "profesional", pero no tenía que ser forzosamente la casa familiar, su formación podía complementarse en los años de adolescencia en la casa de amigos o parientes.

Oakley (1974) identifica cuatro razones que se daban para el confinamiento de la mujer al hogar en la era victoriana (siglo XIX) y que tuvieron repercusiones hasta bien entrado el siglo XX: se condenaba el empleo de la mujer en el terreno de la moral, había la preocupación de que el empleo de la mujer generara un descuido de la salud física y de la familia y, por último, porque contravenía la división "natural" de la labor entre los sexos. Por su parte, Wilson (1991) argumenta, en relación al espacio público, que al convertirse en la provincia masculina la presencia femenina en las calles se vuelve amenazante. Lo que está mal en el diseño de las ciudades, dice, es el deseo masculino de controlar el desorden y, particularmente, la necesidad masculina de controlar el lugar de las mujeres. La explicación que da la autora, para la actitud masculina, es que para los hombres las mujeres de bien deben estar protegidas de los posibles ultrajes que puedan recibir en la calles, por eso mismo aquellas que habitan las calles son mujeres que no tienen ni merecen tal protección, son vistas como un prostitutas, como mujeres en decadencia; la mujer, en este sentido, es pública y la mujer pública se asocia 
con que no es respetable. Durante mucho tiempo, era indecente que una mujer circulara por una calle si no estaba acompañada, y pareciera mantenerse hoy en día, en una variedad de formas, en los países musulmanes y algunos países latinoamericanos. En otras palabras, la mujer era segregada precisamente por no ser considerada respetable cuando ocupa los lugares públicos y de hacerlo, debía adoptar ciertas actitudes: paso regular, cuerpo derecho, sin contoneo, observando donde se pisa para evitar la caída. No era "correcto" fumar, cantar, mirar atrás y se debían evitar los ademanes que pudieran malinterpretarse. Si visitaba lugares públicos como iglesias, debía cubrirse con velo el rostro para librarlo de las miradas (Aristizábal, 2007). Aspectos que pueden coincidir con el reconocimiento que se le dio en la antigüedad (siglos V y VI) al hecho de lograr que los hombres hablen de la mujer lo menos posible, "tanto para alabarla como para criticarla" (Sennet, 2003).

Al revisar los manuales de etiqueta que se crearon en el siglo XIX en Inglaterra y los Estados Unidos, se puede observar que la recomendación principal que se daba a las mujeres era principalmente la de no involucrarse en actividades de la vida pública, por lo que la mejor manera de evitar dificultades en lugares públicos era evitarlos y quedarse en casa. "Si a una mujer se la cae un paquete o un guante al piso y un transeúnte se lo alcanza, la recomendación es sonreír y decir gracias, pero no permitir ninguna conversación adicional" decía uno de los manuales de etiqueta. La intención era la de disminuir el riesgo para las mujeres en la esfera pública (Hutter, 2007, p. 343). En el siglo XIX, se destaca la existencia de documentos como tratados y manuales de urbanidad, que intentan dar pautas para el comportamiento de las mujeres, específicamente en el necesario decoro propio de las señoritas: respeto de sus deberes, recato, conocimiento de los nuevos usos sociales, censura pública y desagrado de la familia. Entre estos documentos, se encuentra el tratado sobre "economía doméstica para el uso de las madres de familia y de las amas de casa" y el manual sobre "breves nociones de urbanidad extractadas de varios autores y dispuestas en forma de catecismo para la enseñanza de las señoritas de la Nueva Granada" (Aristizábal, 2007, p. 148).

En la búsqueda de explicaciones sobre la diferenciación entre lo público y lo privado a partir del género, Chodorow (1978) argumenta que la masculinidad se logra únicamente escapando de la vida domestica. Siendo así, es importante considerar la manera como se distribuye la labor entre hombres y mujeres, pues cuando los hombres no están en el trabajo, están fuera haciendo cosas diferentes, mientras si se trata de las mujeres, cuando no están en el trabajo siguen realizando actividades relacionadas con el hogar, ya sea en su casa o en los lugares públicos: haciendo el mercado, llevando a los niños al colegio y demás actividades afines. Mientras que las mujeres "pertenecen a sus lugares de vivienda", los hombres pueden tener fácilmente acceso a lugares más distantes de su vivienda donde otra gente se reúne. Los lugares públicos para todas las mujeres han sido siempre limitados en la medida en que el espacio de la mujer ha sido fundamentalmente el espacio doméstico, y en público se ha sido limitado a las plazas de mercado, centros comerciales, iglesias y escuelas. Esto es el resultado de la tendencia a dividir los ambientes en sexualmente asimétricos, entre lo privado y lo público, división que contribuye a restringir la movilidad de la mujer en el espacio público y a prevenir su participación como trabajadoras y como ciudadanas.

Los estudios sobre la mujer y el espacio dejan ver la marginalidad de la mujer en el espacio público, pero son pocos los encontrados en la literatura científica en los que se explora la experiencia a partir del rol que desempeña cuando ella hace presencia en el espacio público. Al igual que lo señala Vázquez (1986), la observación sistemática de la vida cotidiana de la mujer en el espacio público urbano, pone de manifiesto la variedad de actividades que ésta realiza y la diversidad de ejes en torno a los que la organizan: el trabajo, la recreación, la vida familiar, etc., que si bien no diferencian de manera excluyente unas categorías de otras, sí denotan tendencias de carácter colectivo. De esta manera, es posible conjeturar que el espacio público es representado y ocupado de forma particular, para distintos roles sociales 
que ocupa la mujer en la sociedad. El estudio que adelantamos recientemente, (Páramo \& Burbano, 2007) con mujeres de distintas edades, condiciones socioeconómicas y distintas actividades sociales, donde se incluyeron: trabajadoras sexuales, vendedoras informales en el espacio público, estudiantes universitarias, ejecutivas trabajadoras de oficina y profesoras de educación básica, exploró los lugares públicos más frecuentados, los usos más frecuentes y la experiencia con los extraños. La investigación muestra evidencia que el rol social que se desempeñe dentro del espacio público, tiene incidencia en la manera como es percibido y usado por las mujeres. Las trabajadoras sexuales y vendedoras informales ven el espacio público, principalmente, como un recurso del cual pueden sacar provecho económico, distinta a la percepción y uso que le dan estudiantes, profesoras y ejecutivas quienes hace de éste, principalmente, un espacio de conectividad para llegar a su destino y menos como lugar de encuentro. Mientras que en las primeras, el contacto con extraños es de tipo comercial, en las segundas es menos frecuente y de mayor desconfianza, a no ser que se trate de lugares públicos en los que se lleve a cabo, de manera ocasional, un acontecimiento cultural que congregue a muchas personas, como es el caso de los conciertos en parques, o una vez por semana, como en las ciclovías de Bogotá. En el caso de las vendedoras informales, se registra un trato amable con los extraños, quizás por el contacto personal y directo con la vendedora, como ocurría antiguamente en las plazas de mercado, situación que ha desaparecido en la actividad comercial, hoy día. La calle es un lugar donde se pueden apreciar las diferencias entre las mujeres; su rol social condiciona no solamente su uso, sino sus percepciones. La relación con otras mujeres extrañas es principalmente de curiosidad, se busca en otras mujeres la moda, el maquillaje, la valoración sobre lo bello y lo feo; quizás sea el espacio público un escenario en el que se transmiten las tendencias de la moda en el vestir. Se hace notar, por parte de quienes participaron en el estudio, que la condición de mujer ofrece algunos "privilegios" que les conceden los hombres en los lugares públicos, cuando se tiene que hacer una fila, pagar un servicio público u ocupar una silla en un transporte público, pero, como mencionamos anteriormente, quizás estas formas de actuar solo están reflejando el control que los hombres quieren ejercer sobre las "mujeres de bien" en los espacios masculinos.

Por último, un reflejo de la ideología masculina sobre el espacio se encuentra igualmente en los principios de diseño y de planeación urbana. Short (1996) acuña el término "ciudad-hechapor-el-hombre" como indicativo de la construcción social del espacio urbano y de la dominación masculina en el diseño y planeación, que refuerza los sesgos de género: los hombres como productores y controladores del espacio y las mujeres como reproductoras de tales estructuras que replican la visión masculinizada del espacio mediante el uso que hacen de éste.

Las calles, parques y en general todos los lugares públicos parecieran igualmente representar la visión masculina de la actividad comercial, deportiva, o recreativa, alejando a las mujeres de estos lugares y haciendo que opten por lugares cerrados como los centros comerciales para hacer compras, el gimnasio para hacer deporte, o el jardín de su casa para entrar en contacto con la naturaleza. Las mujeres en su edad infantil tampoco gozan de las mismas oportunidades para recrearse como sí lo hacen los hombres, pues los espacios propicios para ello, como los parques representan esa visión masculina con que han sido diseñados: juegos construidos en materiales duros diseñados para desarrollar ejercicios que implican mayor esfuerzo (estructuras para monopatín 'skateboarding' y canchas de fútbol) y mobiliario estándar que no reconoce las especificidades de las niñas. Tal vez, esto constituya, en buena parte, la explicación de que ciertos parques son mayormente ocupados por niños que por niñas.

Si bien las cosas han cambiado en las últimas décadas para la mujer, permitiéndole hacer parte de la fuerza laboral, el diseño urbano no ha ido en consonancia con las nuevas responsabilidades que ha asumido. La ubicación de las casas y su diseño desconoce las necesidades de las mujeres para generar ingresos ya sea en el hogar o cerca a él. La presunción de que el trabajo se realiza fuera de la casa 
aumenta la dificultad de las mujeres, para combinar con flexibilidad sus actividades en ambos lugares. El énfasis en el diseño espacial se ha puesto en las actividades laborales consideradas masculinas y, de esta manera, dificultando el desplazamiento que tienen que hacer las mujeres entre su hogar, el colegio de los hijos, los lugares para hacer compras y demás actividades laborales en oficinas ubicadas en el centro de las ciudades, produciendo cruces en horarios e inconveniencias para las mujeres. La única actividad pública que se espera que hagan las mujeres en público es comprar, lo cual también es una extensión de su rol privado como ama de casa. Será por esto que en muchos países los ambientes que son altamente controlados, bien iluminados y bien poblados, hacen de los centros comerciales un lugar seguro y aceptable para las mujeres.

Otra manifestación de la desigualdad en la planeación urbana la vemos en el transporte público. El rol económico de los hombres y sus horarios laborales son la principal preocupación en la localización y programación del tránsito, con menos, si alguna, atención dada al empleo de las mujeres y a los cuidados y responsabilidades del cuidado familiar en su programación diaria. Al igual que los demás componentes de lo que constituye la experiencia en el espacio público, el transporte es visto como neutral respecto al género, asumiendo que beneficia a todos por igual. Por el contrario, el patrón de viaje para algunos, es uno de los aspectos de la vida social con mayor influencia de género (Wachs, 1996). Además de las labores de hogar, ahora la mujer debe desplazarse para dejar a sus hijos en la escuela o algún otro lugar donde tenga que dejarlos, al lugar donde hace el mercado y, por supuesto, al trabajo. El tiempo de estos desplazamientos es mayor y los sistemas de transporte, al igual que el espacio, se han diseñado dentro de la vieja disociación a la que hemos venido haciendo referencia. Por estas razones, las mujeres requieren de horarios más flexibles, mayores conexiones de transporte con centros de servicios, de salud, comerciales, etc. debido, en buena parte, a que es el hombre quien suele hacer uso del automóvil familiar. Al ejercerse mayores demandas para la mujer y complejizarse su situación, le queda poco tiempo para otras actividades lúdicas, sociales o culturales, de ahí que las mujeres conozcan menos las ciudades y se mantengan en mayor aislamiento con respecto a los hombres (Kitchen, 1994; O'Laughlin \& Brubaker, 2007). El tránsito de las mujeres principalmente a pie y en el trasporte público, y el temor al crimen, junto con las medidas precautelares que tiene que tomar cuando se desplaza por el espacio público, restringen el dónde, el cuándo y el cómo se pueden mover en la ciudad. Kuneida y Gauthier (2007) examinan la manera como el género afecta a los usuarios del transporte en las ciudades; las mujeres y los hombres tienen diferentes necesidades de transporte, diferentes comportamientos de viaje y distintos niveles de acceso. Al comparar las mujeres con los hombres en las áreas urbanas, se observa que las mujeres hacen más viajes y más cortos en diferentes momentos del día, lo que tiene implicaciones en tiempo y en dinero. Más aún, las mujeres cabeza de familia tienen diferentes necesidades en la medida en que son responsables de las actividades productivas y reproductivas para el sostenimiento del hogar. Por lo general, las mujeres hacen menos uso de los vehículos particulares y caminan más, lo que refleja las desigualdades en lo niveles de ingreso frente a los hombres. Las mujeres son quienes tienen acceso más tarde a los nuevos sistemas de transporte y los hombres quienes asumen más riesgos con los transportes menos seguros y cuando hay accidentes por cuanto viajan más, pero las mujeres afrontan mayores peligros en su seguridad personal cuando viajan solas. Por otra parte, las mujeres tienen menor acceso al transporte y tienen múltiples propósitos debido a los múltiples roles de trabajo, en el hogar o el cuidado de los niños. Otro aspecto que marca una importante diferencia de género en el transporte, es la seguridad. El hacinamiento en los diferentes sistemas de transporte representa una desventaja adicional para las mujeres, por cuanto facilita el robo y el comportamiento inapropiado de parte de los hombres. Para las mujeres tampoco es seguro viajar en taxi solas.

Domosh y Seager (2001) llaman la atención sobre los lugares de la ciudad a los que las mujeres les temen, entre los que se encuentran además del 
transporte público, las calles y los parques, temores producto de la información que difunden los medios o a las experiencias personales que sufren las mujeres. Independientemente de su rol social, a la mujer se le segrega de los espacios públicos al no permitírsele circular libremente, en la medida en que se invade su privacidad mediante contactos físicos no consentidos, o se le violenta de forma verbal cuando no física. Gardner (1995) hace ver las distintas formas de acoso a las que recurren los hombres para controlar la presencia de las mujeres en los lugares públicos: los chiflidos, silbidos, groserías, pellizcos, contactos físicos, etc. Las mujeres en el espacio público son vulnerables a los ataques corporales y al acoso verbal; son intimidadas con la mirada de los hombres, la presencia de indigentes, enfermos mentales, en su mayoría hombres. El acoso muestra que una vez ellas están en público no acompañadas por hombres, las mujeres no pueden reclamar su derecho a la privacidad como lo pueden hacer los hombres, más aún, en la forma como son miradas o como se comenta acerca de ellas. Molestando a las mujeres en lugares públicos, se evidencia que las mujeres aún son definidas y percibidas en términos de su sexualidad y no gozan del derecho a la privacidad. Más seria aún, es la situación de las trabajadoras sexuales, quienes se exponen permanentemente al acoso de los policías, a violaciones sexuales e insultos. Es el temor a estas agresiones las que se constituyen en el argumento principal para desconfiar de los extraños, de circular por la mayor parte de los lugares públicos, en particular en la noche o por aquellos lugares sucios y solitarios. Pareciera que los hombres no responden a las reglas que ordenan las relaciones sociales, privando a las mujeres y a todos de las prácticas que constituyen el tejido social. Igualmente, la mujer se convierte en una víctima de procesos de zonificación con las tendencias de la ciudad hacia una homogeneidad funcional de tipo social, impidiendo la accesibilidad o señalando lo inapropiado de muchos lugares o muchos servicios públicos vitales para la mujer. Y es esta una de las principales razones por las cuales las mujeres prefieren los centros comerciales a las calles, se sienten más seguras además de la diversidad de estimulación que reciben o distracción: cines, restaurantes, juego para los niños, bares, cafés. Sin embargo, Lofland (1998) argumenta que si bien las mujeres se exponen a peligros al circular por el espacio público, esta situación tiende a exagerarse precisamente para mantenerla fuera de los lugares públicos. Los trabajos de las feministas van en la dirección equivocada al focalizar su atención en el acoso a la mujer, en su seguridad, bienestar y necesidad de protección. Según Lofland (1998) y Wilson (1991) dicha literatura presta mucha atención y expresa hostilidad hacia el espacio público urbano. La violencia contra la mujer ocurre principalmente en el lugar privado de su propia casa. El discurso del terror sobre el espacio público se crea para mantener precisamente a la mujer bajo el control masculino. Con esta forma de actuar lo que se consigue es reforzar la perspectiva paternalista masculina de que la calle es insegura y que los hombres deben ser evitados e ignorados, creándose un círculo que justifica los estereotipos que cada uno tiene del otro (Hutter, 2007). Esta visión antiurbana hace parte de quienes prefieren, por las razones que se han mencionado, ver a la mujer removida del dominio público. Wilson recomienda, por el contrario, una visión más balanceada en la que se muestren los beneficios de hacer visibles a las mujeres, les suministra libertad y oportunidades a la vez que se reconocen los peligros potenciales que hay en la ciudad. No se trata de negar la legitimidad de quienes reclaman una ciudad más segura, libre del acoso y de los peligros a los que se expone la mujer en el espacio público, sino de fortalecer institucionalmente las políticas de policía, para que los transeúntes reciban las consecuencias por no respetar las reglas de convivencia entre géneros (Páramo \& Burbano, 2007).

\section{Conclusión}

Como puede apreciarse por la revisión de la literatura sobre género y ciudad, el espacio público no es neutral. Ha llegado el momento de que los planeadores, gestores urbanos, constructores, Policía, coordinadores de transporte, la ciudadanía en general y los investigadores, dejen de asumirlo de 
esa manera. Con la revisión de la literatura científica sobre las relaciones entre género y espacio y su análisis, pretendemos llamar la atención de los investigadores sobre la necesidad de desarrollar este campo de estudio en el medio latinomericano. No creemos que la falta de estudios que exploren esta relación en nuestro medio, se deba a que no exista un espacio público diferenciado según el género, creemos más bien que la investigación en las disciplinas que estudian la dimensión espacial reproducen la visión masculina, proyectando en sus estudios su sistema de valores en los que lo femenino no representa algo que valga la pena estudiar, o en el peor de los casos algo que no se quiera reconocer.

La revisión del estado del arte sobre la mujer y el espacio público da lugar a formular algunas iniciativas físicoespaciales para el diseño y la planeación del espacio urbano que atiendan las necesidades de las mujeres. Cuando se planifique, se gestione o se establezcan indicadores sobre el espacio público, no debe pensarse de manera abstracta o asumiendo la neutralidad del espacio. Los principios de planeación todavía asumen que el dominio del hombre es lo público, mientras que el de la mujer es lo doméstico y de esa manera el diseño y la planeación de muchos lugares públicos no se ha orientado a atender las necesidades de las mujeres; existe un desconocimiento de sus necesidades espaciales. Hay que señalar que la responsabilidad de esta segregación no solo recae en los individuos particulares, sino también en el Estado al no asumir el diseño espacial para todos, y en las firmas constructoras que no respetan la reglamentación sobre las características que debe tener la obra en cuanto a espacios de sesión, accesibilidad, seguridad, etc. Es importante reconocer que al no respetarse las normas en la cesión que deben hacer los urbanizadores sobre las condiciones de iluminación, amplitud de espacios, zonas verdes, etc. y al ser el Estado permisivo frente a estas irregularidades, se contribuye igualmente, aunque de manera indirecta, a segregar a la mujer del espacio público. A este respecto, se debe contemplar la ampliación y el mejoramiento de andenes y vías (las mujeres se desplazan más caminando que los hombres), el mejoramiento e implementación de accesos a puentes peatonales, mediante un diseño de rampas y escaleras que no demanden mucho esfuerzo para acceder al puente y que las proteja de la mirada de los curiosos. Por otra parte, se hace necesario la construcción de baños públicos por toda la ciudad, bien mantenidos y seguros. Y la recuperación y creación de zonas verdes, con el fin de mejorar las condiciones para el desplazamiento y disfrute de los espacios públicos, que son mayormente utilizados por las mujeres y los niños.

El asunto de la seguridad sigue siendo todavía muy serio con relación al desplazamiento de las mujeres por el espacio público. Alternativas para resolver parte de esta problemática, incluyen, en primer lugar, el reconocimiento de la desigualdad entre las necesidades del hombre y las mujeres, la dicotomía simbólica entre lo público y lo privado, lo masculino con lo femenino, el trabajo con la casa, la suposición de la división de la labor por género. Un aspecto por abordar es la conectividad entre los lugares de vivienda privados y los lugares públicos, adoptando aspectos de diseño y otras medidas para mejorar el tránsito público o el trasporte público e incrementar la seguridad, permitiendo a las mujeres y a los demás viajar más fácilmente, más frecuentemente y durante distintos momentos del día. Buena iluminación, señalización no restringida, eliminación de trampas u oportunidades para asaltos, la habilidad para encontrar ayuda de otros, la visibilidad de otros, son aspectos que deben ser tenidos en cuenta en el diseño del espacio público. La iluminación de las calles y del sistema de transporte, la presencia de policía y la educación ciudadana, son elementos importantes para garantizar el acceso de la mujer al espacio público. Corresponde, entonces, a los programas de cultura ciudadana reconocer que los lugares públicos tienen reglas (Páramo, 2007) y que es necesario enseñarlas, en particular, las de convivencia con las mujeres en el espacio público. 


\section{Agenda de investigación sobre mujer y espacio}

Habrá que investigar otros aspectos relacionados entre la mujer y el espacio público como por ejemplo la edad, la raza, la clase, las preferencias sexuales y la aceptación para ocupar espacios públicos. Las investigaciones sobre las mujeres y la relación entre mujer y ambiente, han identificado igualmente diferencias significativas en las actitudes y actividades de las mujeres de diferentes grupos étnicos y condiciones económicas, aspecto éste que deberá ser estudiado en nuestro medio. El concepto de territorialidad o control ambiental (Páramo, 2007) ofrece perspectivas para estudiar la manera como las mujeres se apropian o territorializan el espacio público. Será necesario, a este respecto, investigar igualmente las distintas necesidades de hombres y mujeres en los equipamientos urbanos que tengan en cuenta los distintos roles sociales.

Se hace necesario preparar a las personas para aceptar la orientación sexual de todas las personas, permitiendo "salir del clóset" tanto a hombres como mujeres que han sido censurados por mostrar sus preferencias en público.

La facilidad de acceso a diferentes tipos de lugares o su proximidad física, espacios públicos seguros o tránsito público, son parte de las necesidades que se han identificado en la literatura sobre las mujeres y el ambiente. En este sentido, nos unimos a los planteamientos que se realizan en el proyecto de investigación denominado La Carta Europea de la Mujer en la Ciudad (s.f.), cuyo propósito es concebir un nuevo planteamiento filosófico de la ciudad, con la intención de contribuir de manera constructiva a un verdadero debate democrático que dé cuenta de las necesidades y de las varias expectativas de los ciudadanos (mujeres y hombres por igual).

Nuestro objetivo ha sido identificar diferencias y aprender como hacer que los ambientes sean más responsivos a las necesidades específicas de las mujeres, que surgen de estas diferencias, y reconocer que la vida cotidiana de las mujeres varía de acuerdo a su rol dentro del lugar público. Esta aproximación incluye estudiar las necesidades que las mujeres tienen, tanto como analizar los ambientes actuales, para determinar si esas necesidades se están satisfaciendo y, de igual manera, formular propuestas alternativas. La observación de las experiencias y percepciones de la mujer sobre el espacio público, dan evidencia a favor de la relatividad del espacio en esta escala urbana y suministra información importante para una planificación y gestión social más equitativa. Los usos del espacio público por parte de las mujeres, sus temores y conflictos con los extraños, se constituyen en un escenario de tensiones entre los elementos de la estructura social, razón de más para no seguir estudiando el espacio de forma absoluta, sino desde su dimensión psicológica y social.

\section{Referencias}

Alarcón, M. (2007). La mujer y los espacios públicos en Bogotá en la primera mitad del siglo XX. Pre-til, 5(13), 58-74.

Aristizábal, M. (2007). Madre y esposa: silencio y virtud. Bogotá: Universidad Pedagógica Nacional.

La Carta Europea de la Mujer en la Ciudad. (s.f.). Recuperado el 30 de enero, 2010, de http://habitat. aq.upm.es/bpn/bp018.html

Chodorow, N. (1978). The reproduction of modering. Berkeley: University of California Press.

Domosh, M. \& Seager, J. (2001). Putting women in place: Feminist geographers make sense of the world. New York: The Guilford Press.

Lofland, L. H. (1998). The public realm: Exploring the city's quintessential social territory. New York: Aldine de Gruyter.

Folguera, P. (1982). La presión del espacio urbano sobre la actividad de la mujer: espacio interior y exterior. Estudios Territoriales, 5, 107-124.

Franck, K. A. (2002). Women and environment. En R. B. Bechtel \& A. Churchman (Eds.), Handbook of Environmental Psychology (pp. 347-362). New York : Wiley.

Gardner, C. B. (1995). Passing by: Gender and public harassment. Berkeley, CA: University of California Press.

Hutter, M. (2007). Experiencing cities. New York: Pearson Education. 
Kitchen, R. (1994). Cognitive maps: What are they and why study them? Journal of Environmental Psychology, 14 (1), 1-19.

Kunieda, M. \& Gauthier, A. (2007). Gender and urban transport: Smart and affordable. Recuperado el 14 de enero, 2010, de http://www.itdp.org/documen ts/7aGenderUT\%28Sept\%29300.pdf

Loyd, B. (1975). Woman's place, man's place. Landscape, 20(1), 10-13.

Oakley, A. (1974). Woman's work: The housewife, past and present. New York: Pantheon Books.

O'Laughlin E. M. \& Brubaker, B. S. (2007). Use of landmarks in cognitive mapping: Gender differences in self report versus performance. Personality and Individual Differences, 24 (5), 595-601.

Páramo, P. (2007). El significado de los lugares públicos para la gente de Bogotá. Bogotá: Universidad Pedagógica Nacional.

Páramo, P. \& Burbano, A. M. (2007). La experiencia de la mujer en el espacio público a partir de su rol social. Pre-til, 5(13), 8-28.

Páramo, P. \& Cuervo, M. (2006). Historia social situada en el espacio público de Bogotá. Bogotá: Universidad Pedagógica Nacional.

Prost, A. \& Vincent, G. (Eds.). (1991). A history of private life: Riddles of identity in modern times (Vol. 5). Cambridge, MA: The Belnap Press.
Saegert, S. \& Winkel, G. (1980). The home: A critical problem for changing sex roles. En G. R. Wekerle, R. Peterson \& D. Morley (Eds.), New space for women (pp. 41-63). Boulder, CO: Westview Press.

Sennet, R. (2003). Carne y piedra. Madrid: Alianza Editorial, S.A.

Short, J. R. (1996). The urban order: An introduction to cities, culture, and power. Cambridge, MA: Blackwell.

Sonnet, M. (1993). La educación de una joven. En G. Duby \& M. Perrot (Eds.), Historia de las mujeres (pp. 142-179). Madrid: Santillana Ediciones Generales, S.L.

Vázquez, A. (1986). Concepción de la mujer: concepción del espacio público. En El uso del espacio en la vida cotidiana. Actas de las IV Jornadas de investigación interdisciplinaria. Seminario de estudios de la mujer. Universidad Autónoma de Madrid.

Wachs, M. (1996). The automobile and gender: An historical perspective. Recuperado el 12 de enero, 2010, de http://www.fhwa.dot.gov/ohim/womens/ chap6.pdf

Wilson, E. (1991). The Sphinx in the city: Urban life, the control of disorder, and women. Barkeley, CA: University of California Press.

Young, I. M. (1990). Throwing like a girl and other essays in feminist philosophy and social theory. Bloominton: Indiana University Press. 